From Fig. 2 it may be noticed that although it is entirely possible to produce a preserved pattern of finite separation both by an accelerated and a decelerated flow

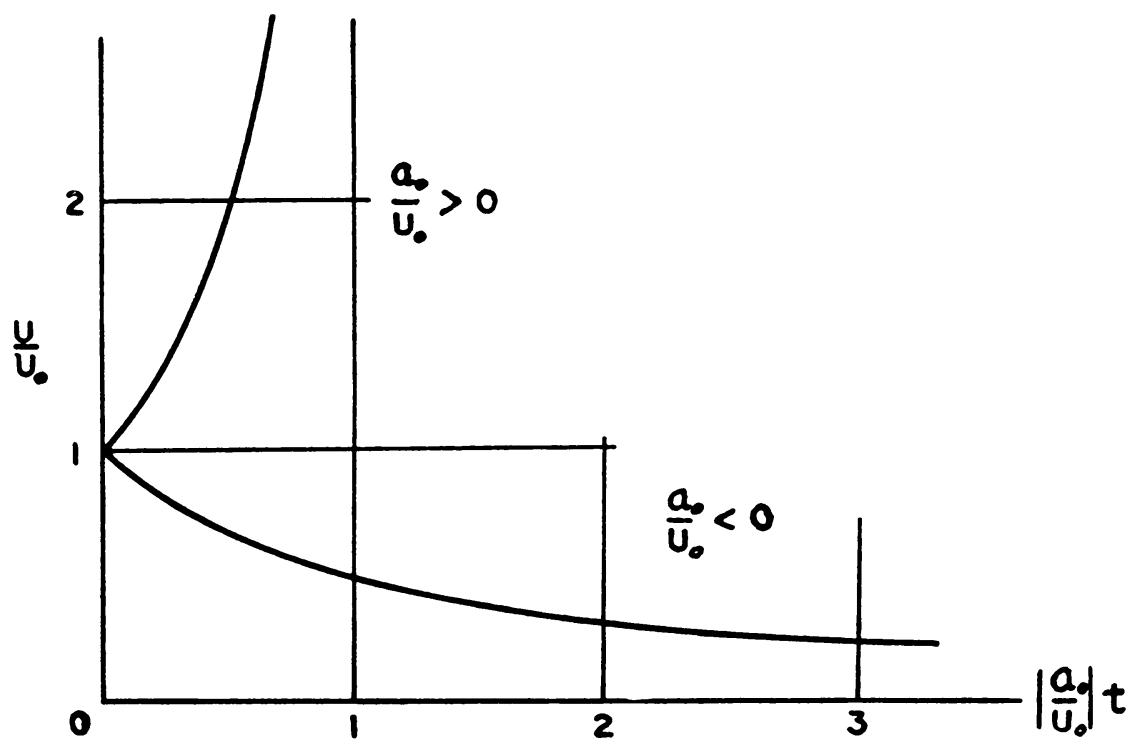

Fig. 2. Variation of flow speed $U$ with time $t$, according to Eq. (7), for accelerated (above) and decelerated (below) flow.

field, yet to maintain such a flow pattern indefinitely, an accelerated field is never adequate; the flow then must be a retarded one.

This analysis definitely rules out the possibility of preserving a finite wake in a stationary flow field, or in any flow field that does not exactly follow the law of motion (7).

\title{
REFERENCES
}

1. L. Prandtl, Uber die Entstehung von Wirbeln in der idealen Flüssigkeit, mit Anwendung auf die Tragfiugeltheorie and andere Aufgaben, pp. 18-33, Vorträge aus dem Gebiet der Hydro-und Aerodynamik, Innsbruck, 1922.

2. T. von Kármán, Accelerated flow of an incompressible fluid with wake formations, Annali Mat. Pur. Appl. (4) 29, 247-249 (1949).

\section{THE BOUNDARY LAYER ON A QUARTER INFINITE FLAT PLATE*}

\section{By L. TRILLING (Massachusetts Institute of Technology)}

This note discusses the incompressible boundary layer on the surface of a quarter infinite flat plate in the absence of a pressure gradient, generalizing the classical two dimensional Blasius solution [1] and Sears' extension to an arbitrarily yawed plate [2]. It shows that the flow retains free stream direction and Blasius profile at all points of the plate, and that the projection of the constant velocity surfaces on planes parallel

*Received May 21, 1953. 
to the plate are curves which become asymptotically parallel to the plate edges far from the lead corner so that the Blasius and Sears solutions are asymptotic cases of the solution given below.

Let the plate occupy the first quadrant of the $X, Y$ plane with the lead corner at the origin; let the free stream velocity vector $U_{\infty}$ be directed along the line $X-Y=0$ (the results obtained in this case can easily be generalized to an arbitrary angle of approach by suitable stretching of the $Y$ coordinate).

The equations satisfied by the flow in the resulting boundary layer are

$$
\begin{aligned}
U_{X}+V_{Y}+W_{z} & =0, \\
U U_{X}+V U_{Y}+W U_{z} & =\nu U_{z Z}, \\
U V_{X}+V V_{Y}+W V_{z} & =\nu V_{z Z},
\end{aligned}
$$

with the boundary conditions

$$
\begin{array}{ll}
U=W=V=0 & \text { on the plate, } \\
U=V=2^{-1 / 2} U_{\infty} & \text { as } \quad Z \rightarrow \infty .
\end{array}
$$

If one seeks solutions of the form suggested by Sears and satisfying the boundary condition (2b), namely with

$$
U(X, Y, Z)=V(X, Y, Z),
$$

Eqs. (1) become

$$
\begin{aligned}
U_{X}+U_{Y}+W_{z} & =0, \\
U\left(U_{X}+U_{Y}\right)+W U_{z} & =\nu U_{z z} .
\end{aligned}
$$

It is convenient to introduce the Blasius parabolic coordinates

$$
x=Z\left(X \nu / U_{\infty}\right)^{-1 / 2}, \quad y=Z\left(Y \nu / U_{\infty}\right)^{-1 / 2},
$$

and the dependent variables

$$
u(x, y)=\frac{2^{1 / 2} U}{U_{\infty}}, \quad w(x, y)=\frac{2^{1 / 2} W Z}{\nu} .
$$

The equations of motion then become, with $r^{2}=x^{2}+y^{2}$,

$$
\begin{gathered}
-r w_{r}+w+\frac{1}{2}\left(x^{3} u_{x}+y^{3} u_{y}\right)=0, \\
w r u_{r}-\frac{u}{2}\left(x^{3} u_{x}+y^{3} u_{y}\right)=r^{2} u_{r r},
\end{gathered}
$$

with the boundary conditions

$$
\begin{aligned}
& u(0)=\frac{w(0)}{r}=0, \\
& \lim _{x, y \rightarrow \infty} u(x, y)=1 .
\end{aligned}
$$


Note that with $y=0, \partial / \partial y=0, r=x$, the flow becomes a Blasius flow since one has

$$
\begin{aligned}
& x w_{x}-w+\frac{1}{2} x^{3} u_{x}=0, \\
& w x u_{x}-\frac{u}{2} x^{3} u_{x}=x^{2} u_{x x} .
\end{aligned}
$$

Differentiating (8b) and substituting for $w, w_{x}$ from $(8 \mathrm{a}, \mathrm{b})$, one obtains

$$
\left(u^{\prime \prime} / u^{\prime}\right)^{\prime}+u / 2=0
$$

which is equivalent to the Blasius equation.

To reduce system $(6 a, b)$ to system $(8 a, b)$ in one variable, one must find a parameter $s(x, y)$ which satisfies the conditions

$$
\begin{gathered}
s \frac{\partial}{\partial s}=r \frac{\partial}{\partial r}=x \frac{\partial}{\partial x}+y \frac{\partial}{\partial y}, \\
s^{3} \frac{\partial}{\partial s}=x^{3} \frac{\partial}{\partial x}+y^{3} \frac{\partial}{\partial y} .
\end{gathered}
$$

In polar coordinates, the condition (9a) is satisfied if

$$
s=r \lambda(\theta),
$$

while (9b) is satisfied if $s=r \lambda(\theta)$ is a solution of

$$
x^{3} s_{x}+y^{3} s_{y}=s^{3}
$$

or, using (10a),

$$
\lambda\left(\cos ^{4} \theta+\sin ^{4} \theta\right)-\lambda^{\prime} \sin \theta \cos \theta=\lambda^{3} .
$$

In terms of the new variables

$$
\alpha=\cos 4 \theta, \quad \mu=\lambda^{2},
$$

Eq. (10c) becomes

$$
\mu(3+\alpha)+2\left(1-\alpha^{2}\right) \mu^{\prime}=4 \mu^{2} .
$$

A particular solution of this equation is

$$
\mu_{0}=(1-\alpha) / 4 .
$$

The general solution can be obtained by substituting

$$
\mu=F(\alpha) \mu_{0}(\alpha)
$$

into (11b). The function $F(\alpha)$ then satisfies the separable equation

$$
2(1+\alpha) F^{\prime}=F(F-1),
$$

so that one has:

$$
F=\left[1-c^{2}(1+\alpha)^{1 / 2}\right]^{-1},
$$


where $C$ is an arbitrary constant. When $C=0$, one finds the solution $\mu_{0}(\alpha)$. Substituting (13c) into (13a) and (11a) and then (10a)

$$
s=(1 / 2) r \sin 2 \theta[1-\alpha \cos 2 \theta]^{-1 / 2} .
$$

Since $s(r, \theta)$ satisfies equations $(10 \mathrm{a}, \mathrm{b}), u(s), w(s)$ satisfy the Blasius Equation $(8 \mathrm{a}, \mathrm{b})$. Writing $s$ in terms of physical coordinates, we have

$$
\begin{gathered}
r=Z\left[U_{\infty}(x+y) / \nu x y\right]^{1 / 2}, \quad \sin \theta=(x / x+y)^{1 / 2}, \quad \cos \theta=(y / x+y)^{1 / 2}, \\
s=2 Z\left(U_{\infty} / \nu\right)^{1 / 2}[(x+y)-a(x-y)]^{-1 / 2} .
\end{gathered}
$$

The constant velocity surfaces are the surfaces $s=$ const. since $u=B l(s)$. It remains to determine the constant $|a|$ in such a way that as $X / Y \rightarrow \infty, s$ becomes proportional to $Z\left(u_{\infty} / \nu y\right)^{1 / 2}$ so that one obtains the Sears yawed plate solution. This is the case if $a=-1,(x>y) ; a=1(x<y)$.

It follows that the present solution extends Sears' result up to the immediate corner of the plate with no change. The constant shear and boundary layer thickness lines are parallel to the axes up to the axis of symmetry. This indicates the existence of a narrow region there where the cross flow terms in the velocity Laplacian are not negligible and the boundary layer equations do not hold.

\section{REFERENCES}

1. H. Blasius, Grenzschichten in Flüssigkeiten mit kleiner Reibung, Zeits. Math. Phys. 56, 1 (1908).

2. W. R. Sears, The boundary layer of yawed cylinders, Journ. Aero. Sci. 15, 49 (1948).

\section{NOTE ON MAXIMUM SHOCK DEFLECTION*}

\section{BY GARRETT BIRKHOFF AND JOHN W. WALSH (Harvard University)}

The angle $\lambda=\lambda(M)$ of maximum shock deflection, for a given Mach number $M$ of flow, is of interest in various applications. It gives the critical angle for attached shocks past a wedge [2, p. 53], and that for jetless wedge collapse [3]. We give here a new simple means of determining $\lambda(M)$, for ideal gases, which seem simpler than the usual one $[1, \S 122]$.

We follow the notation of [2]. By formulas (4.3) and (3.3) of this reference,

$$
q_{2 n}=\frac{a^{* 2}}{q_{1 n}} \quad \text { and } \quad \frac{a^{*^{2}}}{q_{1 n}^{2}}=\frac{\gamma-1}{\gamma+1}+\frac{2}{\gamma+1} \frac{a^{2}}{q_{1 n}^{2}}
$$

in a polytropic gas, with $p+p_{0}=A \rho^{\gamma}$.

Now suppose we are given the velocity $q_{1}$, relative to $J$, of one impinging stream, and the shock angle $\beta$ between the impinging stream and the shock front, as in Fig. 1 . Of course, $\beta$ is not known a priori; we shall seek, by variation of $\beta$, that value of $\beta$ which maximizes the deflection angle $\delta$, and thus obtain the desired maximum deflection $\delta_{\max }=\lambda$.

We suppose also that we know $p$, $\rho$ in the impinging stream. Then the normal shock,

*Received July 15, 1953. 